\title{
Interactions of keV sterile neutrinos with matter
}

\author{
Shin'ichiro Ando ${ }^{1}$ and Alexander Kusenko ${ }^{2,3}$ \\ ${ }^{1}$ California Institute of Technology, Mail Code 350-17, Pasadena, California 91125, USA \\ ${ }^{2}$ Department of Physics and Astronomy, University of California, Los Angeles, California 90095, USA \\ ${ }^{3}$ Institute for the Physics and Mathematics of the Universe, University of Tokyo, Kashiwa, Chiba 277-8568, Japan
}

(Received 28 January 2010; published 18 June 2010)

\begin{abstract}
A sterile neutrino with mass of several $\mathrm{keV}$ is a well-motivated dark-matter candidate, and it can also explain the observed velocities of pulsars via anisotropic emission of sterile neutrinos from a cooling neutron star. We discuss the interactions of such relic particles with matter and comment on the prospects of future direct detection experiments. A relic sterile neutrino can interact, via sterile-active mixing, with matter fermions by means of electroweak currents, with the final state containing a relativistic active neutrino. The recoil momentum impacted onto a matter fermion is determined by the sterile neutrino mass and is enough to ionize atoms and flip the spins of nuclei. While this suggests a possibility of direct experimental detection, we calculate the rates and show that building a realistic detector of the required size would be a daunting challenge.
\end{abstract}

DOI: 10.1103/PhysRevD.81.113006

PACS numbers: 14.60. St, $95.35 .+\mathrm{d}$

\section{INTRODUCTION}

Both cosmology and the supernova physics suggest the possible existence of a sterile neutrino with mass of several $\mathrm{keV}$ [1]. Such a particle could be produced in the early Universe from active-sterile-neutrino oscillations [2-4] or from some other mechanism [5-7] in an amount consistent with the measured dark matter density. The same particle, emitted anisotropically from a cooling neutron star born in a supernova explosion, would cause a neutron star recoil, large enough to explain the observed velocities of pulsars [8-11]. Particle physics models can readily accommodate sterile neutrinos with $\mathrm{keV}$ masses [6,7,12-15], and, in the models with three light sterile neutrinos (dubbed $\nu \mathrm{MSM}$ ), the neutrino oscillations can explain the baryon asymmetry of the Universe [16,17].

The X-ray observations make use of the predicted radiative decay $\nu_{s} \rightarrow \nu_{a} \gamma$ of a sterile neutrino [18,19], which occurs on the time scales much longer than the age of the Universe, but which can yield a non-negligible flux from concentrations of dark matter in astrophysical systems, such as, e.g., galaxies, clusters, and dwarf spheroidal galaxies [1,20-27]. The photons form this two-body decay make a narrow spectral line, broadened only by the velocity dispersion of dark-matter particles. The decay photons produced during the "dark ages" in the early Universe could have affected the formation of the first stars [28-34]. Recent X-ray observations have reported some evidence of the decay line from a sterile neutrino with mass $m_{\nu_{s}} \approx$ $5 \mathrm{keV}$ and the mixing angle squared $\sin ^{2} \theta \approx 10^{-9}$ [27], as well as a $m_{\nu_{s}} \approx 17 \mathrm{keV}$ sterile neutrino with $\sin ^{2} \theta \approx$ $10^{-12}$ [35]. The inferred number densities are consistent with a part or all of dark matter made up of sterile neutrinos. The Lyman- $\alpha$ constraints [36-39] allow up to $40 \%$ of dark matter [40] to be in the form of 5-keV sterile neutrinos produced in the Dodelson-Widrow (DW) sce- nario [2], which generates relatively warm dark matter, while the rest of dark matter is "colder" than the DW component (made up of other species or of sterile neutrinos with the same mass but produced with smaller freestreaming lengths than the DW population). The samemass sterile neutrinos produced by the resonant ShiFuller oscillations [3] make up a colder dark matter than the DW scenario, and the sterile neutrinos produced from the Higgs decays [6,7] are even colder for the same mass $[6,7,41-44]$. Since the warmness of dark matter, measured by the free-streaming length, can vary by a factor $\sim 3$ depending on the production mechanism, the Lyman- $\alpha$ bounds do not preclude that all of dark matter is made up of, for example, 5-keV sterile neutrinos (assuming that DW mechanism is not the dominant mode of production). Apparently, no Lyman- $\alpha$ constraints apply to masses above $10 \mathrm{keV}$ [40], including the $17-\mathrm{keV}$ sterile neutrinos [35]. Moreover, the observations of dwarf spheroidal galaxies appear to show some evidence of warm dark matter, apparently in contradiction with the stated Lyman- $\alpha$ bounds [45]. (For our purposes, it will not make a difference whether sterile neutrinos make up all dark matter or only $40 \%$.)

It is of interest, therefore, to examine the interactions of such particles in matter with the idea of possibly using them for direct detection of relic sterile neutrinos. Unfortunately, the mass and the mixing angle inferred from the X-ray observations, the dark matter abundance, and the pulsar kicks, leave little hope of discovering these particles in neutrino oscillations experiments because the mixing angle squared $\sin ^{2} \theta \approx 10^{-9}$ is too small. The prospects of a nuclear decay experiment with complete kinematic reconstruction proposed in Refs. [46-48] as a means to search for a sterile neutrino are also complicated by the smallness of the mixing angle. 
In this paper we will examine the interactions of relic sterile neutrinos with matter, specifically scattering of sterile neutrinos and matter particles, with the idea of their potential applications to direct detection experiments. Since the initial-state sterile neutrino is nonrelativistic but the final state is a relativistic active neutrino, the kinematics of this scattering differs from that of any other interactions considered in the literature, making this an interesting problem, regardless of its possible applications. The momentum transferred from the sterile neutrino to a target particle is of the order of sterile-neutrino mass ( $p \sim$ $5 \mathrm{keV}$ ), and the kinetic energy imparted to the target electron is, therefore, $T_{e} \sim p^{2} /\left(2 m_{e}\right) \sim 25 \mathrm{eV}$, which is enough to ionize the atoms - a potentially observable feature. This is in contrast with the case of the ordinary relic neutrinos, whose scattering off the matter fermions cannot generate a momentum transfer of more than $10^{-4} \mathrm{eV}$.

If the target particles are polarized in an external magnetic field, then it is also possible that the scattering may flip the spin of the matter fermion, and the return of the spin to its ground state can be accompanied by the emission of a (low-energy) photon. We calculate rates of both these processes, and find that to detect one such event, the exposure of $\sim 1$ yrkton of background-free detector is required. Thus, we conclude that direct experimental detection of relic sterile neutrinos will be very challenging even for the future generation of experiments.

\section{ELECTRON RECOIL AND IONIZATION OF ATOMS}

Let us consider a scattering process between sterile neutrino dark matter and a single electron: $\nu_{s} e^{-} \rightarrow \nu_{e} e^{-}$, which is described by the Feynman diagram in Fig. 1. The kinematically relevant case for the relic sterile neutrinos interacting in matter corresponds to the two nonrelativistic fermions in the initial state. This process is suppressed by mixing angle between sterile neutrinos $\nu_{s}$ and electron

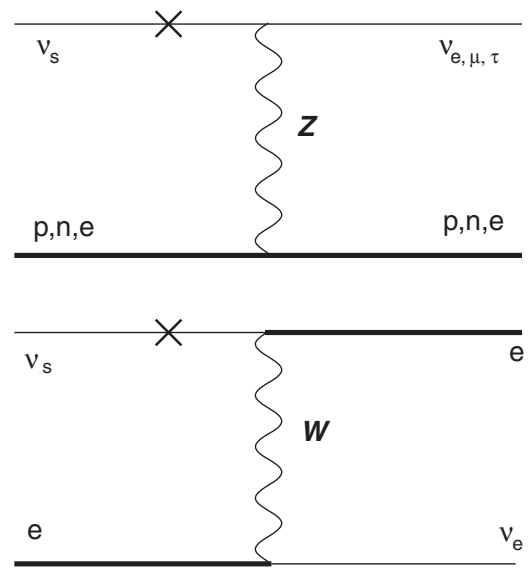

FIG. 1. The Feynman diagrams describing interactions of sterile neutrinos in matter via charged and neutral currents. neutrinos $\nu_{e}, \sin \theta$. Except for this extra suppression factor, the effective Hamiltonian for this process is the same as an ordinary neutrino-electron scattering $\nu_{e} e^{-} \rightarrow \nu_{e} e^{-}$, and it is

$$
\mathcal{H}_{\mathrm{eff}}=\frac{G_{F} \sin \theta}{\sqrt{2}} \bar{\nu}_{e} \gamma_{\mu}\left(1-\gamma_{5}\right) \nu_{s} \bar{e} \gamma^{\mu}\left(c_{V}-c_{A} \gamma_{5}\right) e,
$$

where $c_{V}=1 / 2+2 \sin ^{2} \theta_{W}, \quad c_{A}=1 / 2$, and $\sin ^{2} \theta_{W}=$ 0.23 is the weak-mixing angle. Following the standard procedure, the matrix element squared is evaluated, and it is, after summing over final-state spins and averaging over initial-state spins,

$$
\begin{aligned}
\frac{1}{2} \sum_{\text {spin }}|\mathcal{M}|^{2}= & 4 G_{F}^{2} \sin ^{2} \theta\left[\left(c_{V}+c_{A}\right)^{2}\left(s-m_{e}^{2}\right)\left(s-m_{e}^{2}-m_{\nu_{s}}^{2}\right)\right. \\
& +\left(c_{V}-c_{A}\right)^{2}\left(u-m_{e}^{2}\right)\left(u-m_{e}^{2}-m_{\nu_{s}}^{2}\right) \\
& \left.+2\left(c_{V}^{2}-c_{A}^{2}\right) m_{e}^{2}\left(t-m_{\nu_{s}}^{2}\right)\right]
\end{aligned}
$$

where $s=\left(p_{\nu_{s}}+p_{e}\right)^{2}, \quad t=\left(p_{\nu_{e}}^{\prime}-p_{\nu_{s}}\right)^{2}, \quad u=\left(p_{e}-\right.$ $\left.p_{\nu_{e}}^{\prime}\right)^{2}$ are the Mandelstam variables, and $p_{\alpha}\left(p_{\alpha}^{\prime}\right)$ is the initial-state (final-state) four momentum of a particle $\alpha$.

For evaluating the scattering cross section in a frame where the target electron is at rest, we only leave the leading term in both the matrix element and phase-space integral. In this approximation and for $m_{\nu_{s}} \approx 5 \mathrm{keV} \gg$ $m_{\nu_{e}}$, the momentum transfer is the same as the sterileneutrino mass $\left|\boldsymbol{p}_{e}^{\prime}\right|=\left|\boldsymbol{p}_{\nu_{e}}^{\prime}\right|=m_{\nu_{s}}$, and $\nu_{e}$ is emitted isotropically. The Mandelstam variables are $s \approx m_{e}^{2}+m_{\nu_{s}}^{2}+$ $2 m_{e} m_{\nu_{s}}, t \approx-m_{\nu_{s}}^{2}$, and $u \approx m_{e}^{2}-2 m_{e} m_{\nu_{s}}$. As the result, one obtains the following expression for the scattering cross section:

$$
\begin{aligned}
\sigma_{\nu_{s} e} & =\frac{1}{16 \pi v m_{e}^{2}}\left(\frac{1}{2} \sum_{\text {spin }}|\mathcal{M}|^{2}\right)=\frac{G_{F}^{2} \sin ^{2} \theta}{\pi v} m_{\nu_{s}}^{2}\left(c_{V}^{2}+3 c_{A}^{2}\right) \\
& =7.0 \times 10^{-55} \mathrm{~cm}^{2}\left(\frac{m_{\nu_{s}}}{5 \mathrm{keV}}\right)^{2}\left(\frac{\sin ^{2} \theta}{10^{-9}}\right)\left(\frac{v}{10^{-3}}\right)^{-1},
\end{aligned}
$$

where $v$ is the relative velocity (here the velocity of the dark-matter particle).

Since the momentum transfer to the electron is $\left|\boldsymbol{p}_{e}^{\prime}\right| \approx$ $m_{\nu_{s}}$, the electron kinetic energy in the final state is $T_{e} \approx$ $m_{\nu_{s}}^{2} / 2 m_{e}=25 \mathrm{eV}\left(m_{\nu_{s}} / 5 \mathrm{keV}\right)^{2}$, which is sufficient to ionize the atom. If one can measure the electron spectrum resulting from this interaction, it should peak sharply at $25 \mathrm{eV}$ minus the atomic binding energy. While this is, obviously, a great experimental challenge, the well-defined prediction for the energy spectrum can be useful to reject potential backgrounds.

For the parameters of interest, the scattering of a sterile neutrino off the electrons in an atom is coherent. Because the momentum transfer of the order of $m_{\nu_{s}} \sim 5 \mathrm{keV}$ corresponds to the Compton wavelength on the order of the size of atom, $\sim 10^{-8} \mathrm{~cm}$, the sterile neutrino scatters coherently off all the electrons in the atom. Let us consider an atom of atomic number $Z$ and mass number $A$ as a target. 
Then the scattering cross section with this single atom is

$$
\sigma_{\nu_{s} A}=Z^{2} \sigma_{\nu_{s} e},
$$

which is enhanced by a factor of $\sim 10^{3}$ compared to the single-electron cross section.

Let us now consider the event rate of $\nu_{S} A$ scattering. It is given by $R_{\nu_{s} A}=\sigma_{\nu_{s} A} v n_{\nu_{s}} N_{\mathrm{T}}$, where $n_{\nu_{s}}$ is the number density of sterile neutrino dark matter, and $N_{\mathrm{T}}$ is the number of target atom $A$ in the detector. Assuming that the local mass density of dark matter is $0.4 \mathrm{GeV} \mathrm{cm}^{-3}$ and it is only made of sterile neutrinos, their number density is $n_{\nu_{s}}=8 \times 10^{4} \mathrm{~cm}^{-3}\left(m_{\nu_{s}} / 5 \mathrm{keV}\right)^{-1}$. The number of target atoms is $N_{\mathrm{T}}=\left(6 \times 10^{29} / A\right)\left(M_{\mathrm{det}} /\right.$ ton $)$, where $M_{\mathrm{det}}$ is the mass of a detector. Therefore, the scattering rate is

$$
\begin{aligned}
R_{\nu_{s} A}= & 4.0 \times 10^{-4} \mathrm{yr}^{-1}\left(\frac{m_{\nu_{s}}}{5 \mathrm{keV}}\right)\left(\frac{\sin ^{2} \theta}{10^{-9}}\right) \\
& \times\left(\frac{M_{\mathrm{det}}}{1 \mathrm{ton}}\right)\left(\frac{Z}{25}\right)^{2}\left(\frac{A}{50}\right)^{-1} .
\end{aligned}
$$

According to Eq. (5), a kton-scale detector can expect about one interaction per year due to dark-matter sterile neutrinos. Detecting a spectral line of $25 \mathrm{eV}$ energy electrons is a formidable challenge in view of the various backgrounds. The relic sterile neutrinos could also interact with nuclei via the neutral currents. However, these interactions give nuclear recoils of negligible energy. Still, the same interaction flips a spin of a nucleus in an external magnetic field in a magnetic resonance experiment, as we detail in the next section.

\section{SPIN FLIP OF A NUCLEUS}

The interaction cross section [Eq. (3)] does not depend on the target electron mass. As the electron is always nonrelativistic, the same expression should also be applicable to the sterile-neutrino-nuclei scattering. (We note, however, the values of $c_{V}$ and $c_{A}$ are different, because of internal structure of nuclei as well as absence of chargedcurrent interaction.) Although the kinetic energy imparted to a nucleus is not sufficient to yield detectable signal, the flip of nuclear spins due to the interaction might be observed, if they are initially aligned in an external magnetic field, similar in some sense to the nuclear magnetic resonance experiments.

One might expect that part of the axial-current term depending on $c_{A}^{2}$ of Eq. (3) is the spin-flip cross section, and it is indeed true. In this section, we show this explicitly in the case of scattering between sterile neutrino and spin-1/2 nuclei for simplicity. We follow the notations of Ref. [49].

To leading order, it is enough to regard sterile neutrinos and target nuclei as nonrelativistic and take their fourcomponent spinors as $u=\sqrt{m}(\xi, \xi)^{T}$, where $m$ is the particle mass and $\xi$ is the corresponding two-component spinor. On the other hand, the final-state (left-handed) neutrino is relativistic, so the spinor is given as $u_{\nu_{e}}=$ $\sqrt{2 E_{\nu_{e}}}\left(\xi_{\nu_{e}}, 0\right)^{T}$ with neutrino energy $E_{\nu_{e}}$. We choose the $z$-axis in the direction of incident $\nu_{s}$, and $\theta_{\nu}$ denotes the scattering angle of the final-state neutrino $\nu_{e}$. Then the two-component spinors of neutrinos are $\xi_{\nu_{s}}=(0,1)^{T}$ and $\xi_{\nu_{e}}=\left(-\sin \left(\theta_{\nu} / 2\right), \cos \left(\theta_{\nu} / 2\right)\right)^{T}{ }^{1}$ The initial-state nucleus is assumed to have spin up along direction $\left(\theta_{s}, \phi_{s}\right)$, where $\theta_{s}$ is the angle between $z$-axis and spin axis and $\phi_{s}$ is the azimuthal angle measured from the plane of scattering. Then $\xi_{N}(\uparrow)=\left(\cos \left(\theta_{s} / 2\right), e^{i \phi_{s}} \sin \left(\theta_{s} / 2\right)\right)^{T}$. For the spin-flip process, the final-state nucleus has spin down along the same direction: $\xi_{N}(\downarrow)=$ $\left(-e^{-i \phi_{s}} \sin \left(\theta_{s} / 2\right), \cos \left(\theta_{s} / 2\right)\right)^{T}$.

The spin-flip matrix element is

$$
\begin{aligned}
i \mathcal{M}(\uparrow \rightarrow \downarrow)= & -\frac{i G_{F} \sin \theta}{\sqrt{2}} \bar{u}_{\nu_{e}}\left(p_{\nu_{e}}^{\prime}\right) \gamma_{\mu}\left(1-\gamma_{5}\right) u_{\nu_{s}}\left(p_{\nu_{s}}\right) \\
& \times \bar{u}_{N}^{\downarrow}\left(p_{N}^{\prime}\right) \gamma^{\mu}\left(c_{V}-c_{A} \gamma_{5}\right) u_{N}^{\dagger}\left(p_{N}\right) \\
= & -4 i G_{F} \sin \theta c_{A} m_{N} \sqrt{E_{\nu_{e}} m_{\nu_{s}}} \\
& \times\left(2 \sin \frac{\theta_{\nu}}{2} \cos ^{2} \frac{\theta_{s}}{2}-e^{i \phi_{s}} \cos \frac{\theta_{\nu}}{2} \sin \theta_{s}\right) .
\end{aligned}
$$

It is straightforward to take the absolute value squared of Eq. (6), but one must average over the directions of the incident sterile neutrinos, that is over the relative angle between $z$-axis and spin, $\left(\theta_{s}, \phi_{s}\right)$. After taking the average over this angle, the matrix element squared is

$$
\overline{|\mathcal{M}(\uparrow \rightarrow \downarrow)|^{2}}=16 G_{F}^{2} \sin ^{2} \theta c_{A}^{2} m_{N}^{2} m_{\nu_{s}} E_{\nu_{e}}\left(1-\frac{\cos \theta_{\nu}}{3}\right) .
$$

One must integrate over the phase space. We assume that, in an external magnetic field $B$, the spin up state is the ground state and the spin down state is the excited state, while the energy difference is given by $2 \mu_{N} B$, where $\mu_{N}$ is the magnetic moment of the nuclei. In the limit of infinitely heavy nuclei, the neutrino energy is given by $E_{\nu_{e}} \approx m_{\nu_{s}}-$ $2 \mu_{N} B$. Since $m_{\nu_{s}}$ is in the $\mathrm{keV}$ scale, it is always possible to neglect $\mu_{N} B$ in the above expression, for any realistic values of $B$. Thus the kinematics of the scattering is the same as the case of $B=0\left(E_{\nu_{e}} \approx m_{\nu_{s}}\right.$ and neutrinos are emitted isotropically), and we have the same phase-space integral as before. Therefore, for the spin-flip cross section, we obtain

$$
\begin{aligned}
\sigma(\uparrow \downarrow) & =\frac{1}{32 \pi v m_{N}^{2}} \int_{-1}^{1} d \cos \theta_{\nu} \overline{|\mathcal{M}(\uparrow \rightarrow \downarrow)|^{2}} \\
& =\frac{G_{F}^{2} \sin ^{2} \theta}{\pi v} m_{\nu_{s}}^{2} c_{A}^{2},
\end{aligned}
$$

a similar expression as the axial-current term in Eq. (3).

\footnotetext{
${ }^{1}$ Thanks to the active-sterile mixing, the left-handed component is part of the "sterile" mass eigenstate (suppressed by the small mixing angle).
} 
Although $B$ does not appear in the cross section, the magnetic field is important to arrange the directions of nuclear spins, and it can be adjusted such that the transition from excited to ground state emits photons of appropriate wavelength. The degree of polarization of the initial state also depends on the temperature.

As the sterile neutrino changes the nuclear spin, there is no $Z^{2}$ coherence enhancement in this process. Therefore, the event rate is even smaller than the case of ionization of atoms. Still, it is perhaps worth keeping this interaction in mind, since it is not clear which of these processes come out to be experimentally feasible in the far future.

We note that the cross section for opposite transition $\sigma(\downarrow$ $\rightarrow \uparrow)$ is the same as Eq. (8). It is also straightforward to show that the total cross section $\sigma(\uparrow \rightarrow \uparrow)+\sigma(\downarrow \rightarrow \downarrow)+\sigma(\uparrow$ $\rightarrow \downarrow)+\sigma(\downarrow \rightarrow \uparrow)$ is given by Eq. (3).

\section{DECAY OF STERILE NEUTRINOS}

Finally, relic sterile neutrinos can decay inside the detector volume into the lighter neutrino and an the X-ray photon via reaction $\nu_{s} \rightarrow \gamma \nu_{a}$ [18]. The radiative decay width is equal to $[18,19]$

$$
\begin{aligned}
\Gamma_{\nu_{s} \rightarrow \gamma \nu_{a}} & =\frac{9}{256 \pi^{4}} \alpha_{\mathrm{EM}} G_{F}^{2} \sin ^{2} \theta m_{\nu_{s}}^{5} \\
& =\frac{1}{1.8 \times 10^{21} \mathrm{~s}} \sin ^{2} \theta\left(\frac{m_{\nu_{s}}}{\mathrm{keV}}\right)^{5} .
\end{aligned}
$$

For the local dark-matter density, this process would produce $4 \times 10^{-9}$ decays per cubic meter per year. Obviously, in a terrestrial lab, the interactions of sterile neutrinos with liquid or solid matter occur much more frequently than decays in the same volume. Of course, the decays produce $\mathrm{X}$-rays, which can ionize hundreds of atoms per decay.

\section{DISCUSSION AND CONCLUSIONS}

We have examined the interactions of relic sterile neutrinos in matter, and we have calculated the rates of such interactions, assuming that dark matter is comprised of sterile neutrino with mass $\sim 5 \mathrm{keV}$. The interactions of such particles with the electrons can give the electrons a
$25-\mathrm{eV}$ kinetic energy, which is enough to ionize the atom, but the expected rates of interactions are as low as one per kiloton per year, even if the scattering cross section is coherently enhanced. A scattering off a nucleus can flip the nuclear spin. The cross section of this process is given by a similar expression (if nuclear spin is $1 / 2$ ) as that of scattering with electrons, and the rate is also very small.

In addition to small interaction rates, an experiment would face the challenge of rejecting the backgrounds, which, in general, depend on the experimental techniques employed for detection. One feature of our signal that can help in background rejection is a narrow width of the 25$\mathrm{eV}$ line, $\Delta E / E \sim 10^{-3}$. If the experiment can measure the direction of the electron recoil, it can be used to reject the solar neutrino background. The flux of solar neutrinos at the relevant energies is $\sim 10^{8} \mathrm{~cm}^{-2} \mathrm{~s}^{-1}$, with a power-law spectrum, and their arrival directions are determined by the position of Sun. The flux of $5 \mathrm{keV}$ sterile neutrinos must be $F \sim 3 \times 10^{12} \mathrm{~cm}^{-2} \mathrm{~s}^{-1}$ if they are to account for the local dark matter density $\rho_{\mathrm{DM}} \sim 0.4 \mathrm{GeV} \mathrm{cm}^{-3}$, and their interactions (suppressed by the mixing angle) produce a narrow spectral line in the spectrum of the recoil electrons. If the experiment can distinguish between solar and antisolar directions, it will be able to reject the solar background.

As for the experimental techniques, several proposals that have been put forth for detecting relic active neutrinos [50-52] could be useful for an admittedly futuristic detector [53]. Such proposals include interactions with free electrons, tritium beta decay, superconductors, transition edge sensors, and interactions with nanometer size granules [52-54]. Although, at present, neither of these proposals seems to provide the basis for a feasible experiment capable of detecting relic sterile neutrinos, one can hope that an opportunity may arise in the future.

\section{ACKNOWLEDGMENTS}

The work of S. A. was supported by Japan Society for Promotion of Science. The work of A. K. was supported by DOE Grant No. DE-FG03-91ER40662 and NASA ATFP Grant No. NNX08AL48G.
[1] A. Kusenko, Phys. Rep. 481, 1 (2009).

[2] S. Dodelson and L. M. Widrow, Phys. Rev. Lett. 72, 17 (1994).

[3] X.-D. Shi and G. M. Fuller, Phys. Rev. Lett. 82, 2832 (1999).

[4] T. Asaka, M. Laine, and M. Shaposhnikov, J. High Energy Phys. 01 (2007) 091.

[5] M. Shaposhnikov and I. Tkachev, Phys. Lett. B 639, 414 (2006).

[6] A. Kusenko, Phys. Rev. Lett. 97, 241301 (2006).
[7] K. Petraki and A. Kusenko, Phys. Rev. D 77, 065014 (2008).

[8] A. Kusenko and G. Segrè, Phys. Lett. B 396, 197 (1997).

[9] G. M. Fuller, A. Kusenko, I. Mocioiu, and S. Pascoli, Phys. Rev. D 68, 103002 (2003).

[10] M. Barkovich, J. C. D'Olivo, and R. Montemayor, Phys. Rev. D 70, 043005 (2004).

[11] A. Kusenko, B. P. Mandal, and A. Mukherjee, Phys. Rev. D 77, 123009 (2008).

[12] T. Asaka, S. Blanchet, and M. Shaposhnikov, Phys. Lett. B 
631, 151 (2005).

[13] T. Asaka, M. Shaposhnikov, and A. Kusenko, Phys. Lett. B 638, 401 (2006).

[14] F. Bezrukov, H. Hettmansperger, and M. Lindner, Phys. Rev. D 81, 085032 (2010).

[15] G. B. Gelmini, E. Osoba, and S. Palomares-Ruiz, Phys. Rev. D 81, 063529 (2010).

[16] E. K. Akhmedov, V. A. Rubakov, and A. Y. Smirnov, Phys. Rev. Lett. 81, 1359 (1998).

[17] T. Asaka and M. Shaposhnikov, Phys. Lett. B 620, 17 (2005).

[18] P. B. Pal and L. Wolfenstein, Phys. Rev. D 25, 766 (1982).

[19] V. D. Barger, R. J. N. Phillips, and S. Sarkar, Phys. Lett. B 352, 365 (1995).

[20] K. Abazajian, G. M. Fuller, and M. Patel, Phys. Rev. D 64, 023501 (2001).

[21] K. Abazajian, G. M. Fuller, and W. H. Tucker, Astrophys. J. 562, 593 (2001).

[22] A. D. Dolgov and S. H. Hansen, Astropart. Phys. 16, 339 (2002).

[23] A. Boyarsky, A. Neronov, O. Ruchayskiy, M. Shaposhnikov, and I. Tkachev, Phys. Rev. Lett. 97, 261302 (2006).

[24] C. R. Watson, J. F. Beacom, H. Yuksel, and T. P. Walker, Phys. Rev. D 74, 033009 (2006).

[25] H. Yuksel, J.F. Beacom, and C.R. Watson, Phys. Rev. Lett. 101, 121301 (2008).

[26] M. Loewenstein, A. Kusenko, and P. L. Biermann, arXiv:0812.2710.

[27] M. Loewenstein and A. Kusenko, Astrophys. J. 714, 652 (2010).

[28] P.L. Biermann and A. Kusenko, Phys. Rev. Lett. 96, 091301 (2006).

[29] J. Stasielak, P. L. Biermann, and A. Kusenko, Astrophys. J. 654, 290 (2007).

[30] J. Stasielak, P.L. Biermann, and A. Kusenko, Acta Phys. Pol. B 38, 3869 (2007).

[31] J. Stasielak, P. L. Biermann, and A. Kusenko, arXiv:astro$\mathrm{ph} / 0701585$.
[32] M. Mapelli, A. Ferrara, and E. Pierpaoli, Mon. Not. R. Astron. Soc. 369, 1719 (2006).

[33] E. Ripamonti, M. Mapelli, and A. Ferrara, Mon. Not. R. Astron. Soc. 375, 1399 (2007).

[34] M. Valdes, A. Ferrara, M. Mapelli, and E. Ripamonti, Mon. Not. R. Astron. Soc. 377, 245 (2007).

[35] D. A. Prokhorov and J. Silk, arXiv:1001.0215.

[36] M. Viel, J. Lesgourgues, M. G. Haehnelt, S. Matarrese, and A. Riotto, Phys. Rev. D 71, 063534 (2005).

[37] U. Seljak, A. Slosar, and P. McDonald, J. Cosmol. Astropart. Phys. 10 (2006) 014.

[38] M. Viel et al., Phys. Rev. Lett. 100, 041304 (2008).

[39] A. Boyarsky, J. Lesgourgues, O. Ruchayskiy, and M. Viel, J. Cosmol. Astropart. Phys. 05 (2009) 012.

[40] A. Boyarsky, J. Lesgourgues, O. Ruchayskiy, and M. Viel, Phys. Rev. Lett. 102, 201304 (2009).

[41] D. Boyanovsky, Phys. Rev. D 77, 023528 (2008).

[42] K. Petraki, Phys. Rev. D 77, 105004 (2008).

[43] D. Boyanovsky, Phys. Rev. D 78, 103505 (2008).

[44] J. Wu, C.-M. Ho, and D. Boyanovsky, Phys. Rev. D 80, 103511 (2009).

[45] R.F.G. Wyse, 9th UCLA Symposium on Sources and Detection of Dark Matter and Dark Energy in the Universe, Marina del Rey, 2010 (to be published).

[46] G. Finocchiaro and R. E. Shrock, Phys. Rev. D 46, R888 (1992).

[47] F. L. Bezrukov and M. Shaposhnikov, Phys. Rev. D 75, 053005 (2007).

[48] D. Gorbunov and M. Shaposhnikov, J. High Energy Phys. 10 (2007) 015.

[49] M.E. Peskin and D. V. Schroeder, An Introduction to Quantum Field Theory (Addison-Wesley, Reading, MA, 1995).

[50] N. Cabibbo and L. Maiani, Phys. Lett. 114B, 115 (1982).

[51] P. Langacker, J. P. Leveille, and J. Sheiman, Phys. Rev. D 27, 1228 (1983).

[52] G. B. Gelmini, Phys. Scr. t121, 131 (2005).

[53] P. F. Smith, Ann. N.Y. Acad. Sci. 647, 425 (1991).

[54] P. F. Smith and J. D. Lewin, Phys. Lett. 127B, 185 (1983). 